\title{
Magnetic Quasi-phase Matching All-Fiber Isolator
}

\author{
Chunte A. Lu and Gerald T. Moore \\ Advanced Electric Laser Branch, Air Force Research Laboratory, 3550 Aberdeen Ave SE, Kirtland Air Force Base, \\ Albuquerque, NM 87117, USA
}

Correspondence should be addressed to Chunte A. Lu, chunte.lu@kirtland.af.mil

Received 2 December 2009; Accepted 1 March 2010

Academic Editor: Samir K. Mondal

Copyright ( $\odot 2010$ C. A. Lu and G. T. Moore. This is an open access article distributed under the Creative Commons Attribution License, which permits unrestricted use, distribution, and reproduction in any medium, provided the original work is properly cited.

We have experimentally demonstrated an all-fiber optical isolator with $20 \mathrm{~dB}$ isolation. The result shows that the quasi-phase matching technique via a meter-long magnet array is highly feasible to generate more than 45 degrees of Faraday rotation in the fibers. The all-fiber isolator can also be temperature tuned to operate between $1048 \mathrm{~nm}$ and $1066 \mathrm{~nm}$ wavelength.

\section{Introduction}

While ytterbium- (Yb-) doped fiber laser amplifiers have reached multi-kW power level, the power limit of available fiber-coupled isolators is only around $50 \mathrm{~W}$. Free-space bulk isolators have capability of handling higher power; however, it is inconvenient and highly inefficient to recouple light back into the fiber. To our best knowledge, there are no monolithic all-fiber isolators available for the $\mathrm{Yb}$ emission band. Without appropriate isolators to prevent back reflection and the build-up of backward-travelingstimulated Brillouin scattering, backward amplification is capable of causing catastrophic failure of high-power laser systems.

\section{All-Fber Isolator Design and Fiber PBL Measurements}

Our objective is to build an all-fiber isolator based on Faraday rotation in fused silica fiber [1]. The all-fiber isolator setup is shown in Figure 1. With the high magnetic field of Neodymium (NdFeB) magnets, we expect to obtain $45^{\circ}$ Faraday rotation within the wavelengths of the $\mathrm{Yb}$ emission band using an array of magnets close to $1 \mathrm{~m}$ long. The magnets are mounted in two rows in a repelling configuration, such that the direction of the longitudinal field in the gap between the rows reverses periodically. A specialty fiber (Nufern low birefringence PM fiber) is inserted in the gap to produce the desired Faraday rotation, and its polarization beat length $(\mathrm{PBL})$ is matched to the period of the magnet array. Due to this quasi-phase matching, laser emission entering the magnet array in one eigen-polarization of the fiber exits with equal amounts of the two eigenpolarizations [2, 3]. To demonstration an isolator, all-fiber polarizers are spliced to either ends of the birefringent fiber. The input polarizer is oriented in the direction of the eigenpolarizations; the polarization axis of the output polarizer is positioned at $45^{\circ}$ with respect to the eigen-polarizations. The fiber is translated longitudinally to obtain linear polarization at $45^{\circ}$ at the position of the latter polarizer. One major advantage of this setup is that more than one fiber can be inserted in the magnet gap to produce multiple isolators.

We first purchased the low-bi Nufern PANDA fiber and then specified the period of the magnet array to match the measured PDL of the fiber at $1064 \mathrm{~nm}$. We investigated three different types of fiber PBL measurement techniques: polarization modulation in the spectra of broadband light transmitted through the fiber with and without point stress [4], Rayleigh scattering of narrow-band light out the side of the fiber, and Faraday rotation of narrow-band light induced by passing the fiber through a small hole in a magnet that is translated along the fiber. The broadband point-stress measurement shows the most decisive result. The measurements were made for 8 stress points spaced by 5 $\mathrm{mm}$. The broadband source was coupled into the low-bi fiber by bulkhead coupling. The output analyzer is oriented at $45^{\circ}$ 


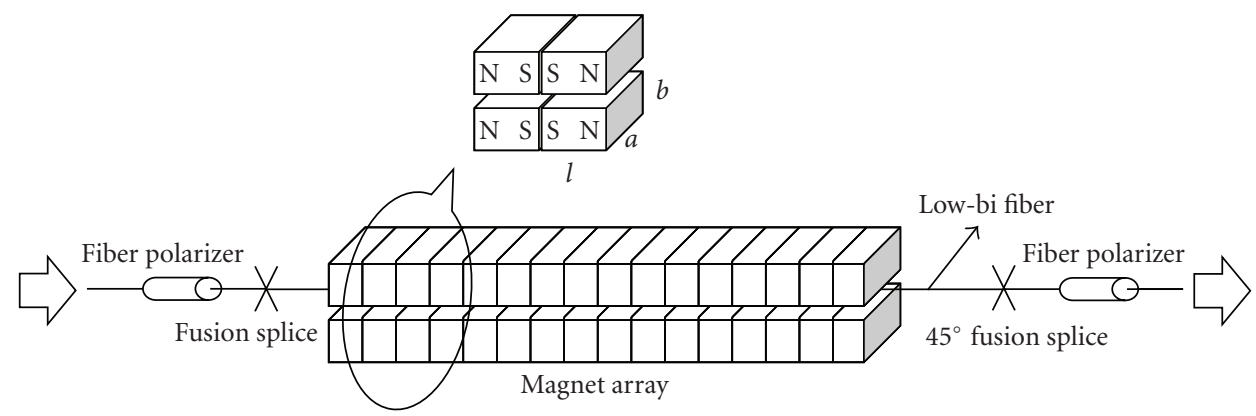

Figure 1: All-fiber isolator setup. Phase matching condition for producing Faraday rotation of light in a fiber within the gap is when the fiber PBL equals $2 l$.

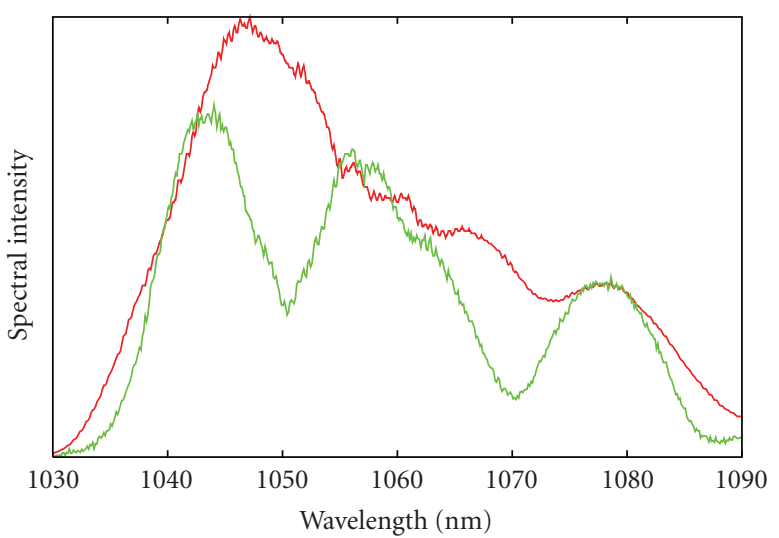

(a)

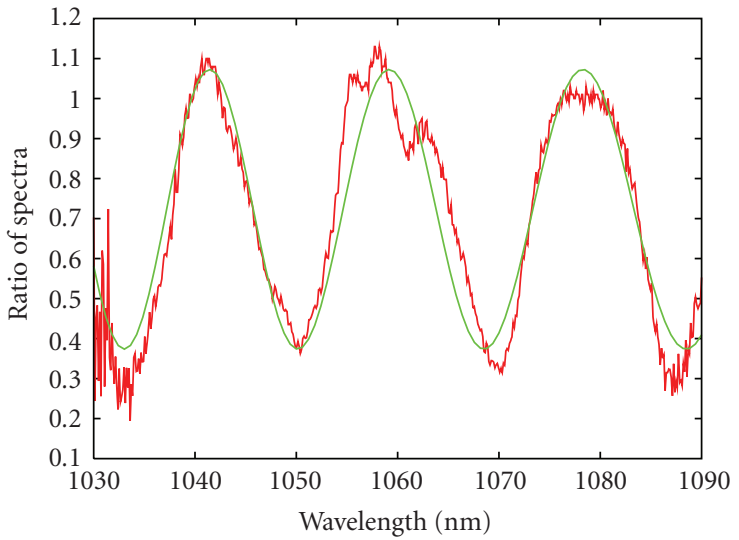

(b)

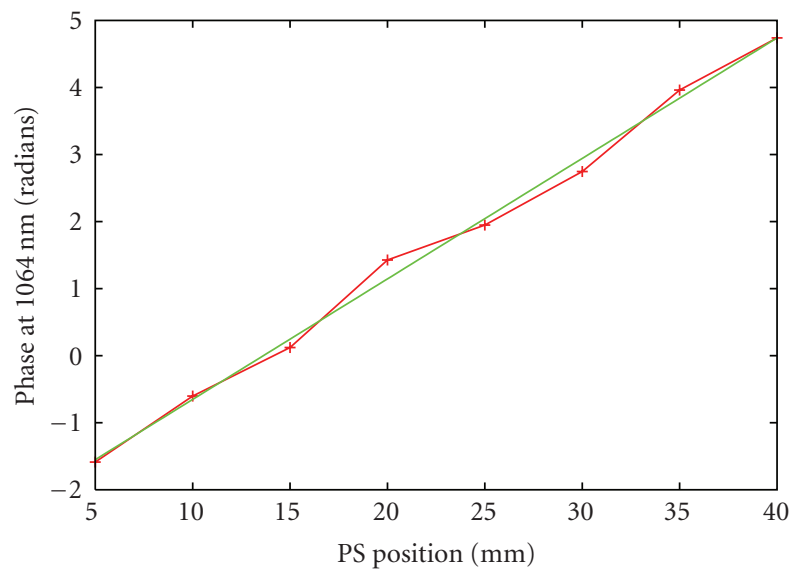

(c)

Figure 2: (a) shows an example of spectrum without (red) and with (green) application of a point stress. (b) shows the ratio of the spectra (red) and the fitting (green) to a $\cos ^{2}$ function. (c) shows the phase (red) of the $\cos ^{2}$ function at $1064 \mathrm{~nm}$ for 8 positions of the point stress and a fit to a straight line (green).

with respect to the slow axis of the fiber. The experimental data and analysis are shown in Figure 2.

Dividing $\pi$ by the slope of the fitted straight line in Figure 2(b) gives the PBL. This PBL applies only to the region where the point stress was applied and does not prove that the PBL is uniform over the whole fiber. If for the purpose of estimation we assume that the fiber PBL is uniform, we can then calculate the total phase advance from the point stress to the end of the fiber and determine the wavelength dependence of the PBL shown in Figure 3.

We chose a magnet period of $1.75 \mathrm{~cm}$ for demonstrating an optical isolator near $1064 \mathrm{~nm}$. We anticipated that some wavelength or temperature tuning would be required to achieve ideal phase matching. The Verdet constant of silica glass determines that the magnet array needs to have about 57 periods (228 total magnets) to produce $45^{\circ}$ Faraday 
rotation in the fiber. To satisfy the phase matching condition for a $1.75 \mathrm{~cm}$ fiber PBL and to obtain a sufficiently strong magnetic field, the dimensions of each individual magnet are specified to be $a=24 \mathrm{~mm}, b=12 \mathrm{~mm}$, and $l=8.75 \mathrm{~mm}$.

\section{Experiments and Results}

Although the preliminary point stress broadband PBL measurement gives a reasonable idea of the fiber PBL, the actual PBL of the fiber at $1064 \mathrm{~nm}$ and room temperature is close to $1.78 \mathrm{~cm}$. This is observed when we phase matched the magnet array and fiber at $1045 \mathrm{~nm}$ and room temperature. Based on experimental thermal study of the low-bi fiber at 20,25 , and $30^{\circ} \mathrm{C}$ and the phase matching result, we obtained the relationship between fiber PBL, wavelength, and temperature shown in Figure 4. To increase the phase matching wavelength to around $1060 \mathrm{~nm}$, a decrease in temperature of $10^{\circ} \mathrm{C}$ or more is necessary.

Phase matching at $1060 \mathrm{~nm}$ wavelength is verified experimentally with a broadband source when the fiber is cooled to $10^{\circ} \mathrm{C}$. Polarized light is injected along the slow axis of the low-bi fiber with the fiber positioned at the center of the magnet gap. The output is first coupled through a polarization analyzer and then into a spectrum analyzer. By translating the fiber in the longitudinal direction of the magnet array, quasi-phase matching is observed and shown in Figure 5. Each graph represents normalized spectra when the analyzer is oriented at $45^{\circ}$ and $90^{\circ}$ with respect to the fiber axes and the fiber is translated $2 \mathrm{~mm}$ between each frame. The experimental data (red and green curves) is fitted with theoretical analysis (blue and pink curves) in each measurement.

Because birefringence in the fiber causes optical power in each eigen-polarization to be maintained outside the magnet array, the measured output spectrum is independent of the longitudinal fiber translation when the analyzer is oriented at $0^{\circ}$ or $90^{\circ}$. The wavelengths where $45^{\circ}$ Faraday rotation occurs are where the spectra with the $90^{\circ}$ output analyzer (normalized to the sum of the $0^{\circ}$ and $90^{\circ}$ spectra) cross the 0.5 transmission line. The spectra with the $45^{\circ}$ output analyzer (normalized to the sum of the $+45^{\circ}$ and $-45^{\circ}$ spectra) have transmissions close to zero or unity at the wavelengths where the Faraday rotation is $45^{\circ}$, provided that the fiber is translated to inject linearly polarized light into the analyzer. As more fiber is translated out of the magnet array, the transmission spectra shift to higher wavelengths until the next PBL period starts.

We demonstrated an all-fiber isolator by splicing fiber polarizers made by Victor [5] onto the Nufern low-bi 10/125 PM fiber used for Faraday rotation. The input polarizer is aligned with the axes of the low-bi fiber, while the output polarizer is rotated by $45^{\circ}$. The isolator is tested using a low-power broadband source which could be coupled into either ends of the isolator, and the spectrum of the light emerging from the opposite end is monitored. The low-bi fiber is positioned in the magnet array so as to minimize backward transmission at a wavelength near phase matching, and the forward transmission is observed at the same fiber position.

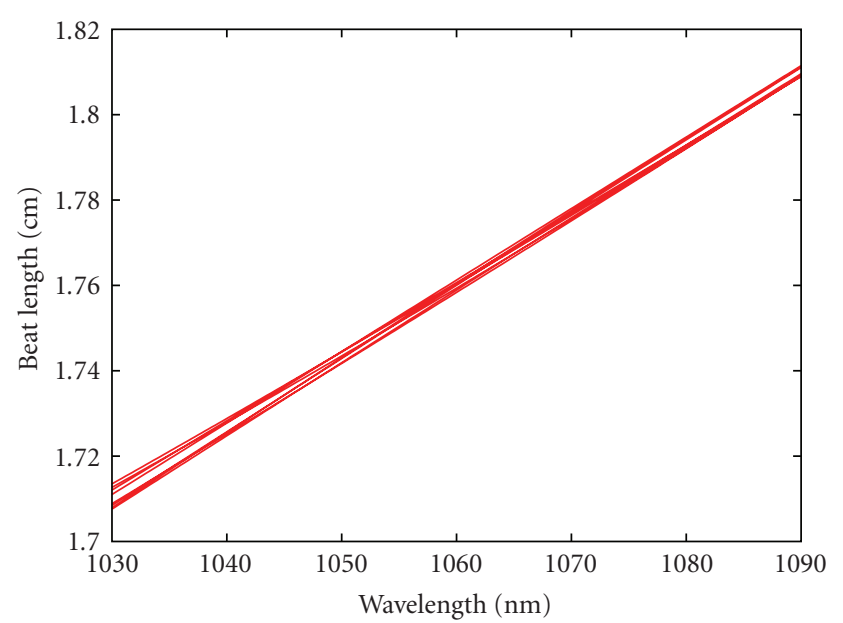

FIGURE 3: Fiber PBL as a function of wavelength as determined for each of 8 stress points. The graph shows a fiber PBL of above 1.76 at $1064 \mathrm{~nm}$ wavelength. A series of such measurements gave values $1.748,1.726,1.763,1.739,1.811,1.824,1.800,1.741,1.826,1.769$, and $1.752 \mathrm{~cm}$.

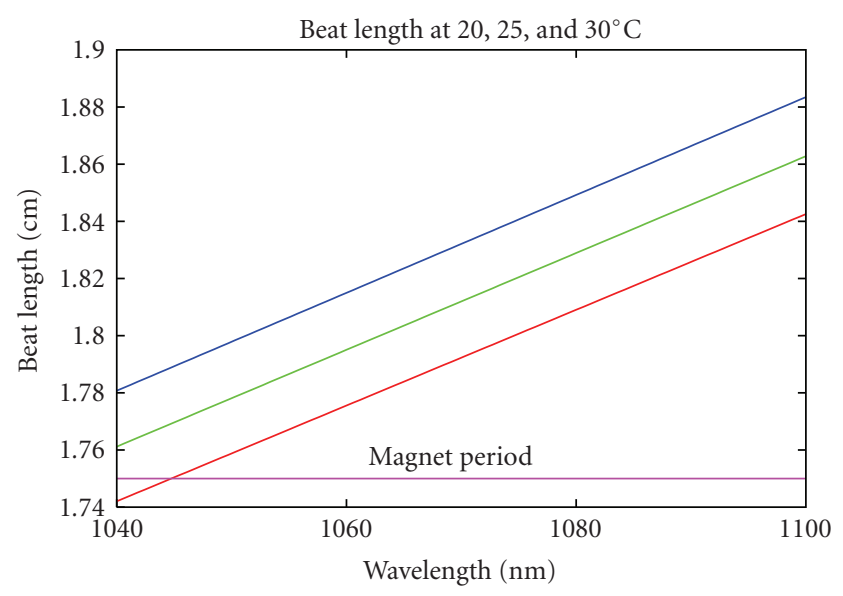

FIGURE 4: PBL with wavelength and temperature dependents where red curve is for $20^{\circ} \mathrm{C}$ and blue is for $30^{\circ} \mathrm{C}$. Phase matching can be achieved at $1060 \mathrm{~nm}$ when the temperature of the fiber is at $10^{\circ} \mathrm{C}$.

The graphs below show the spectra of the quasimatched all-fiber isolator at room temperature and at $5^{\circ} \mathrm{C}$. The data is normalized by equalizing the forward and backward transmission far from phase matching, where there is little Faraday rotation and one expects 50\% transmission in either direction. We observe good isolation $(\sim 20 \mathrm{~dB})$ near $1048 \mathrm{~nm}$ at room temperature and $1066 \mathrm{~nm}$ at $5^{\circ} \mathrm{C}$. The high-frequency oscillating structure in the spectra might result from imperfect polarization of the broadband source and its transmission to the isolator through high-bi PM fiber.

\section{Summary}

We have demonstrated a $20 \mathrm{~dB}$ isolation all-fiber isolator with a broadband source at $1048 \mathrm{~nm}$ and $1066 \mathrm{~nm}$. In the current setup, both the maximum power handling capability 


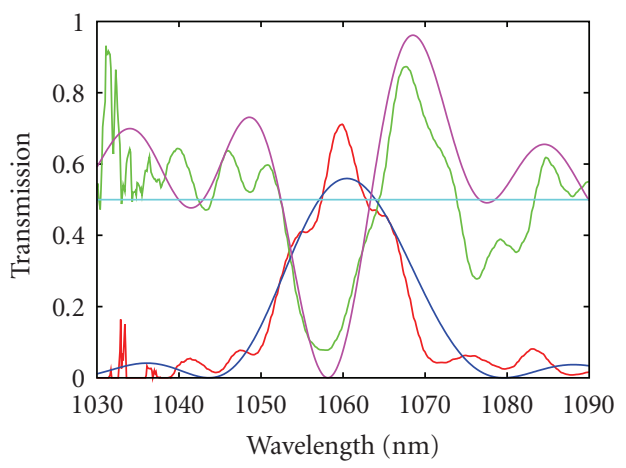

(a)

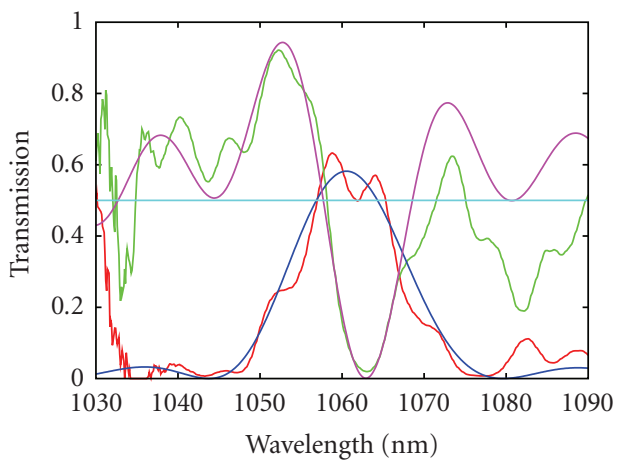

(c)

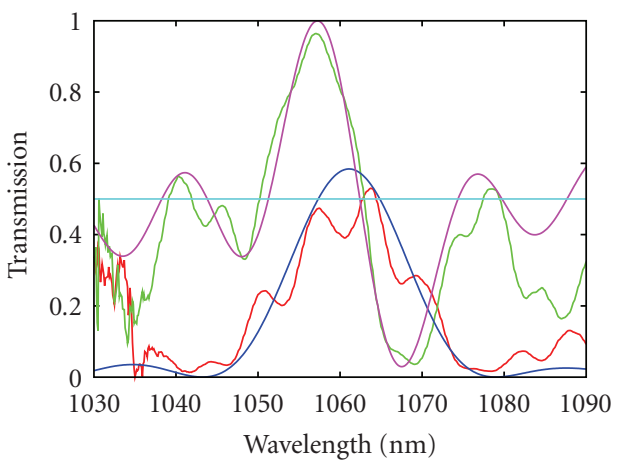

(e)

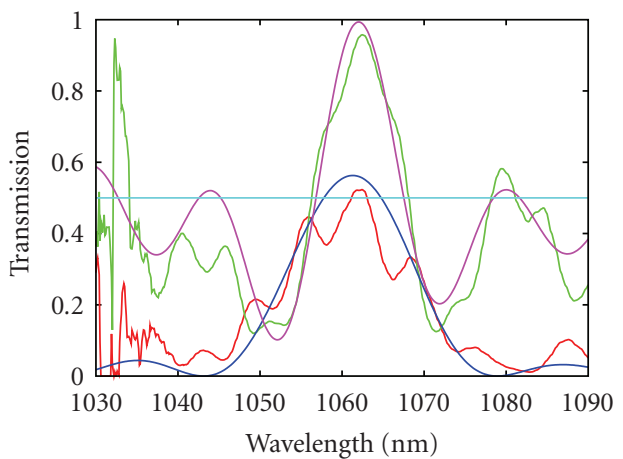

(g)

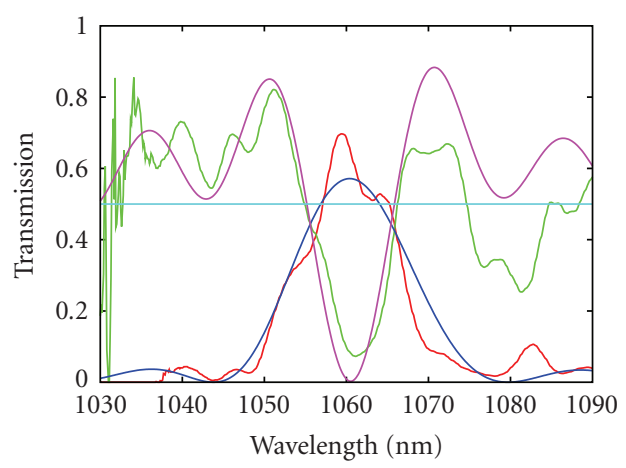

(b)

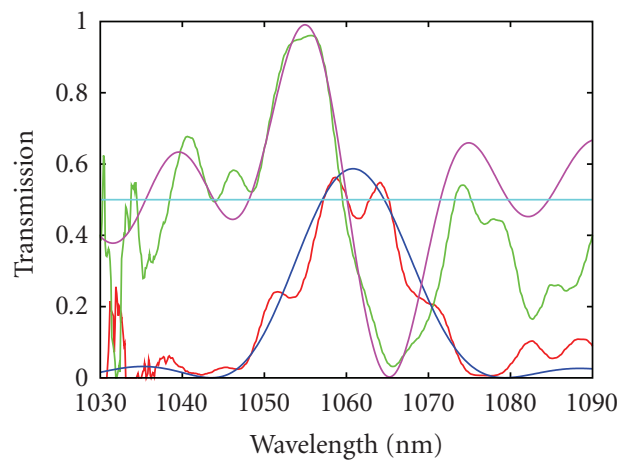

(d)

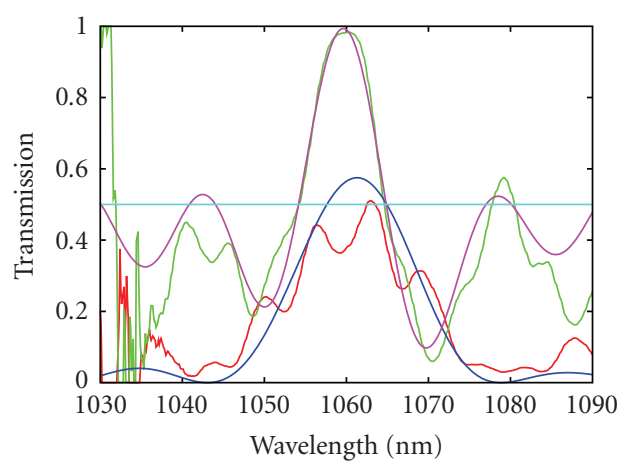

(f)

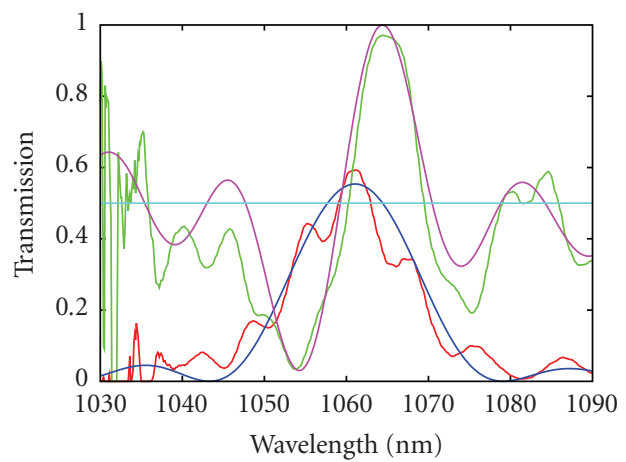

(h)

FIGURE 5: Quasi-phase matching for $1060 \mathrm{~nm}$ wavelength at $10^{\circ} \mathrm{C}$. Red and green curves are experimental observations when the output analyzer is oriented at $90^{\circ}$ and $45^{\circ}$, respectively. Blue and pink curves are theoretical fittings of the spectra. The graphs that show more than $45^{\circ}$ Faraday rotation is achieved around $1060 \mathrm{~nm}$. 


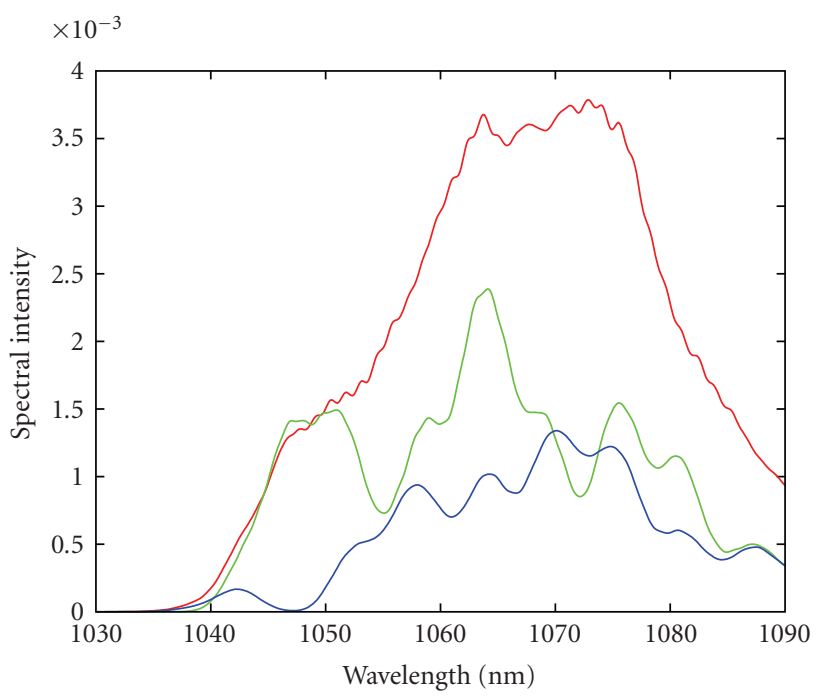

(a)

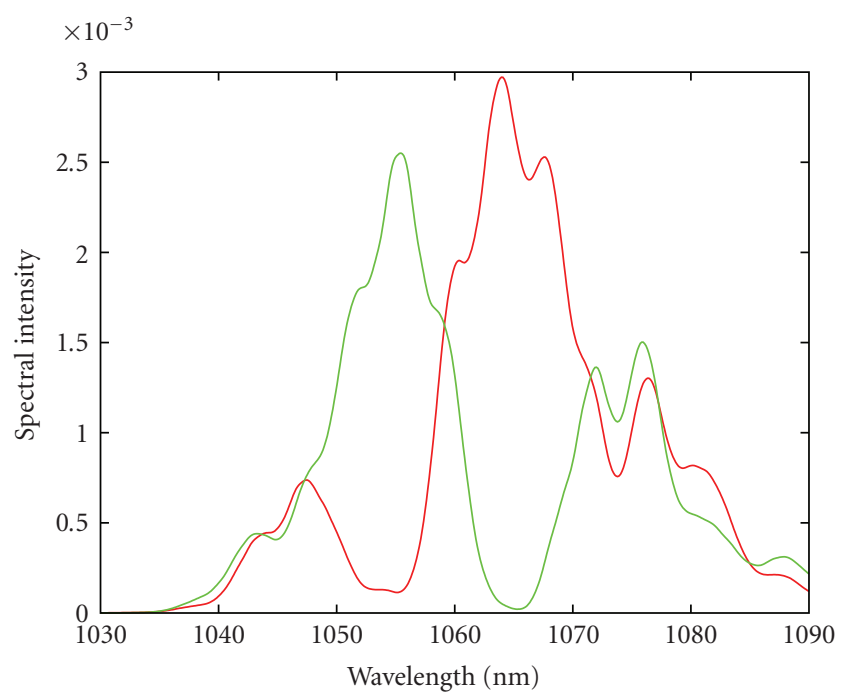

(b)

FIGURE 6: (a) shows normalized spectra of the ASE source (red), forward direction (green), and backward direction (blue) at room temperature $\left(\sim 20^{\circ} \mathrm{C}\right)$. (b) shows normalized forward (red) and backward (green) spectra when the fiber is cooled to $10^{\circ} \mathrm{C}$.

and degree of isolation are limited by the fiber polarizer, which is in the order of $10 \mathrm{~W}$ and $20 \mathrm{~dB}$, respectively. The insertion loss of the Chiral fiber polarizer (which has a smaller core size than the low-bi fiber) is about $1 \mathrm{~dB}$. This is high compared to typical free-space polarizers, but it does allow us to verify the all-fiber isolator concept. In future work we plan to try $45^{\circ}$ tilted fiber Bragg grating polarizers [6], and we are currently investigating their insertion loss, polarization-extinction ratio, and power limitations. We expect that the tilted grating has higher power handling capability and extinction ratio than our current fiber polarizer. We are also planning to dope the silica fiber with high Verdet constant material such as terbium ( $\mathrm{Tb})[7]$ to reduce the size of the isolator and simplify the temperature tuning mechanism. The quasi-phase matched all-fiber isolator concept will also be implemented with fiber containing both solid and with liquid stress elements $[8,9]$. We expect the tunable birefringence of this fiber to aid in developing future high-power isolators.

\section{Acknowledgment}

The authors would like to acknowledge financial support from the High Energy Laser-Joint Technology Office (HEL-JTO).

\section{References}

[1] J. L. Cruz, M. V. Andres, and M. A. Hernandez, "Faraday effect in standard optical fibers: dispersion of the effective Verdet constant," Applied Optics, vol. 35, no. 6, pp. 922-927, 1996.

[2] E. H. Turner and R. H. Stolen, "Fiber Faraday circulator or isolator," Optics Letters, vol. 6, no. 7, pp. 322-324, 1981.

[3] G. T. Moore, "In-fiber optical isolator for high-power operation," US patent no. 7,336,858, February 2008.

[4] P. Hlubina and D. Ciprian, "Spectral-domain measurement of phase modal birefringence in polarization-maintaining fiber," Optics Express, vol. 15, no. 25, pp. 17019-17024, 2007.

[5] V. I. Kopp, V. M. Churikov, and A. Z. Genack, "Synchronization of optical polarization conversion and scattering in chiral fibers," Optics Letters, vol. 31, no. 5, pp. 571-573, 2006.

[6] K. Zhou, G. Simpson, X. Chen, L. Zhang, and I. Bennion, "High extinction ratio in-fiber polarizers based on $45^{\circ}$ tilted fiber Bragg gratings," Optics Letters, vol. 30, no. 11, pp. 1285-1287, 2005.

[7] L. Sun, S. Jiang, J. D. Zuegel, and J. R. Marciante, "Effective Verdet constant in a terbium-doped-core phosphate fiber," Optics Letters, vol. 34, no. 11, pp. 1699-1701, 2009.

[8] G. T. Moore, "Optical fiber with tunable birefringence using pressurized liquid capillaries," US patent no. 7,489,847, February 2009.

[9] K. Nielsen, D. Noordegraaf, T. Sørensen, A. Bjarklev, and T. P. Hansen, "Selective filling of photonic crystal fibres," Journal of Optics A, vol. 7, no. 8, pp. L13-L20, 2005. 

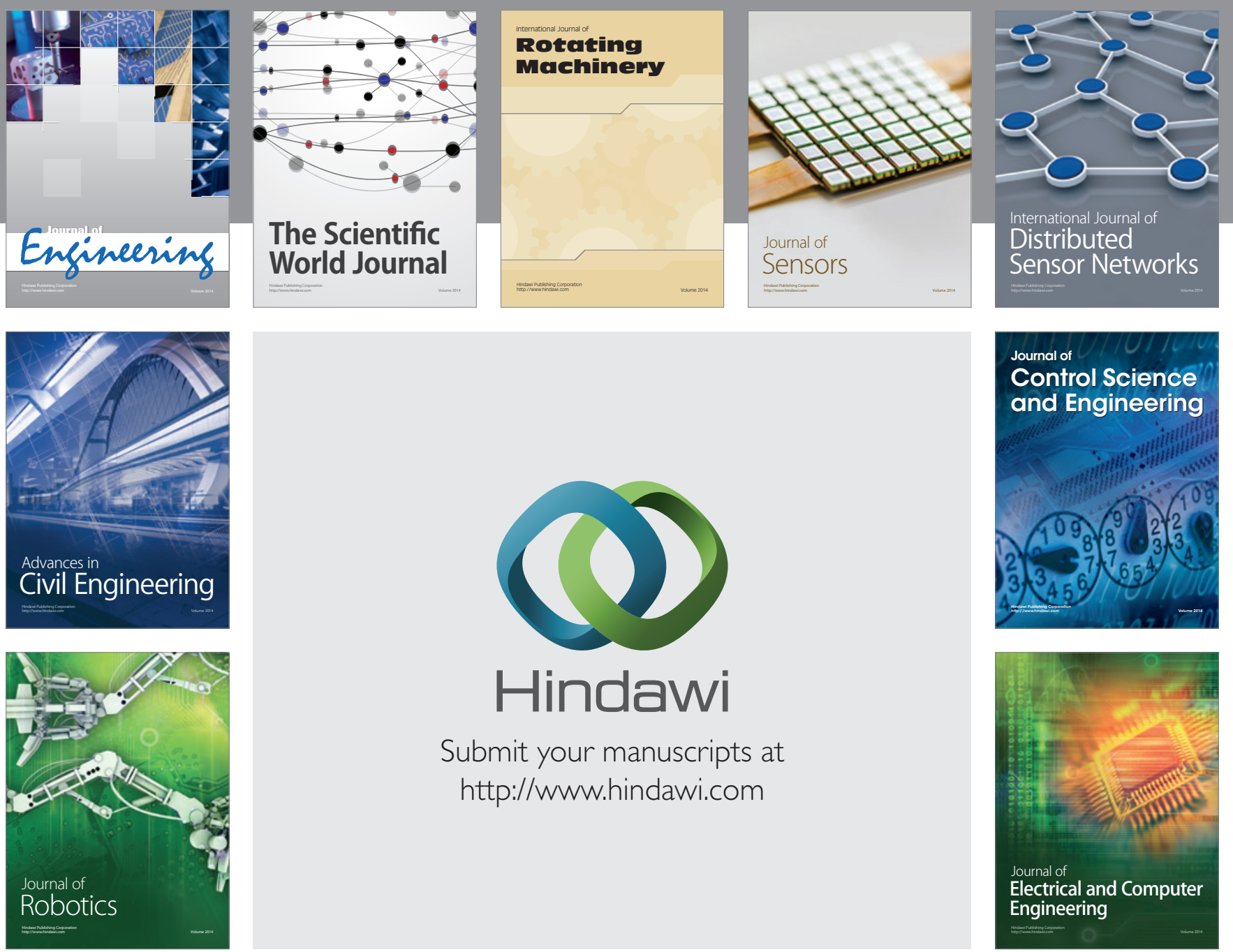

Submit your manuscripts at

http://www.hindawi.com
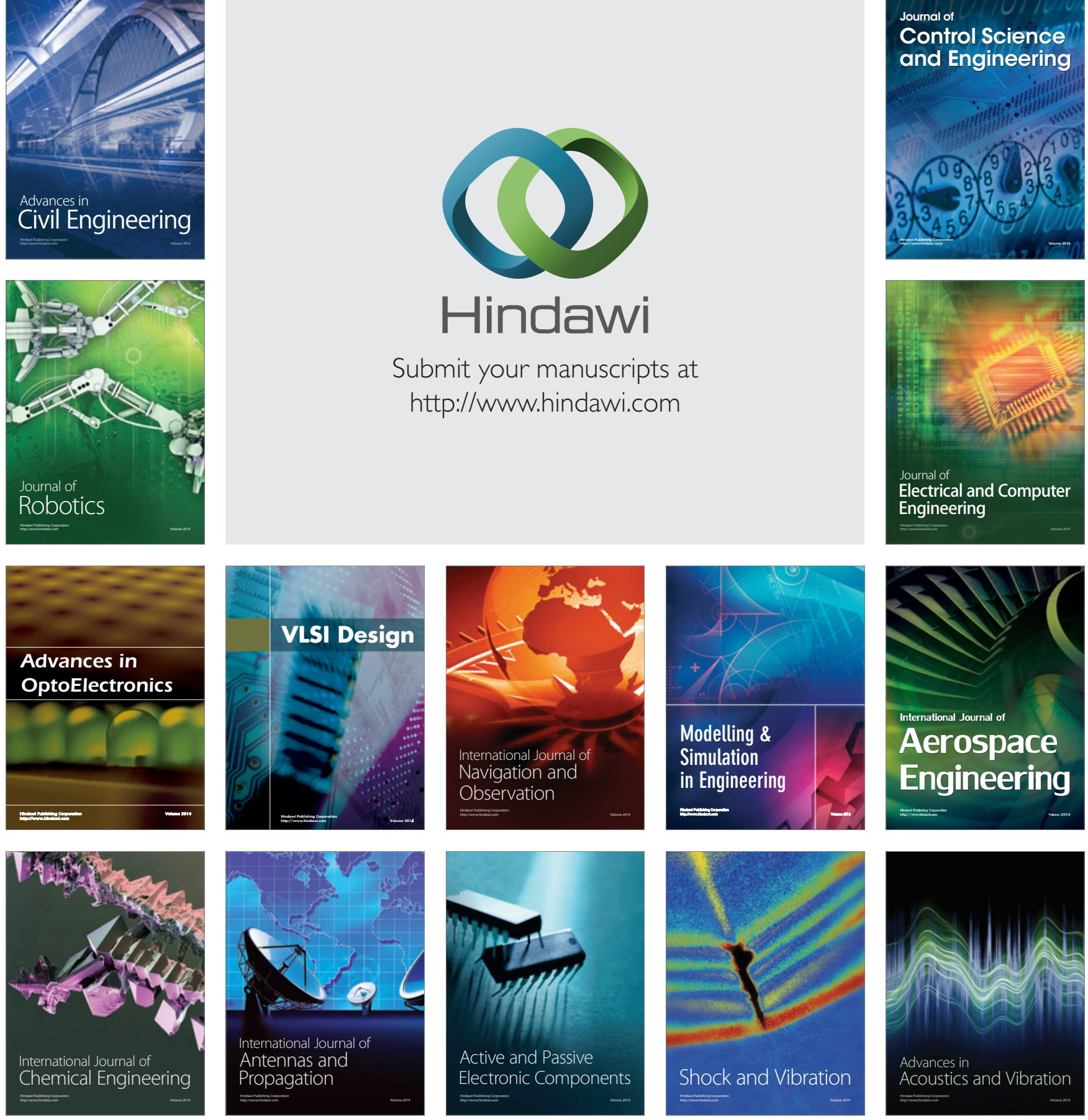